\title{
OTIOTOMICS
}

Revista de economía, empresa y sociedad

Dossier "Claves para entender el turismo de hoy"

EN BUSCA DE UN MODELO MÁS EFICIENTE

\section{Hacia una nueva gobernanza del destino turístico: el enfoque de gestión de los destinos turísticos inteligentes (DTI) 1}

\section{David Giner Sánchez}

Profesor colaborador de los programas de turismo de la UOC. Instituto Valenciano de Tecnologías Turísticas (Invat.tur)

RESUMEN La evolución de las TIC e Internet muestra una clara influencia directa e indirecta en todos los ámbitos de desarrollo de los destinos turísticos, tanto en tareas internas como externas. Los destinos afrontan un nuevo ciclo de crecimiento en el que deben apoyarse, con mayor o menor intensidad, en el uso de nuevos desarrollos tecnológicos, pero también en nuevas fórmulas y estrategias de gestión y planificación. Se trata, al fin y al cabo, de abordar con garantías la necesidad de reorientarse hacia un modelo más eficiente, adaptado a los cambios de la demanda y basado en la integración de todos los elementos que conforman el destino, el turista y la población local entre ellos.

Es en este contexto en el que surge un nuevo marco de gestión en cuya base aparece la gobernanza turística y donde el uso de las TIC e Internet se presenta como pieza clave para facilitar esa transición hacia el nuevo modelo de destino turístico inteligente, basado en el conocimiento y en la participación activa de todos sus agentes, entre otros.

PALABRAS CLAVE gobernanza turística; TIC; innovación; destinos inteligentes

\section{Towards a new governance of tourist destinations: a focus on the management of smart tourism destinations}

\begin{abstract}
The evolution of ICTS and the internet has a clear influence on all areas of tourist destination development, both directly and indirectly, and in both internal and external tasks. Destinations face a new cycle of growth, one in which they must take advantage - to varying degrees - of the use of new technological developments as well as new formulae and strategies for management
\end{abstract}

1. Este trabajo se enmarca en el proyecto de investigación «Nuevos enfoques para la planificación y gestión del territorio turístico: conceptualización, análisis de experiencias y problemas. Definición de modelos operativos para destinos turísticos inteligentes» (Proyecto CSO2014-59193-R) del Programa Estatal de I+D+I del Ministerio de Economía y Competitividad. 
and planning. Essentially, the challenge is to tackle with assurance the need for reorientation towards a model that is more efficient, one that is adapted to changes in demand, and is based on an integration of all the elements making up the destination, the tourists and the local population.

In this context there emerges a new framework for management, a basic feature of which is the governance of tourism, and in which ICTs and the internet are key factors in the transition to the new model of smart tourism destinations. An important part of the basis for this framework will be knowledge and the active participation of all agents.

\section{KEYWORDS tourism governance; ICT; innovation; smart destinations}

\section{Introducción: la revolución tecnológica y su influencia en la gestión de los destinos turísticos}

Los destinos se enfrentan a retos complejos para su gestión y desarrollo; por eso tienen que dar respuesta a una serie de necesidades de los turistas y del sector turístico, así como de la población residente, negocios locales y otras industrias vinculadas (Howie, 2003). Por tal razón, la gestión de los destinos desempeña un papel clave en la orientación de la mayoría de las cuestiones, en ocasiones conflictivas, que surgen derivadas del desarrollo turístico en la actualidad (Crouch y Ritchie, 1999). Todo ello exige una correcta planificación y gestión del destino desde todos los puntos de vista.

Al tiempo que han evolucionado los modelos de gestión de los destinos turísticos, es evidente que también lo ha hecho el propio concepto de destino turístico desde el punto de vista teórico, reflejando así muchos de los cambios que han ido incorporándose en sus modelos y estructura de gestión. La evolución de las relaciones entre los diferentes agentes ha supuesto el principal elemento generador de cambios en el concepto y la estructura de gestión de los destinos turísticos. Los estudios e investigaciones sobre turismo se han centrado en el análisis del concepto de destino desde múltiples orientaciones que se pueden resumir en las siguientes (Saraniemi y Kylänen, 2011): económico-geográfica, gestión de marketing y de cliente.

Mientras que la orientación económico-geográfica y la de marketing hacen referencia al papel del consumidor para situarlo como destinatario de la experiencia turística, la referida al enfoque de cliente pone al turista en el eje de la creación del producto turístico del destino. Para Mosisander y Valtonen (2006), el turista se considera un consumidor pasivo cuando es una parte más de las acciones que desarrollan los proveedores de servicios turísticos, tal y como proponen las anteriores orientaciones. Pero, en cambio, también señalan que el turista puede considerarse un cliente activo cuando participa en la creación de la experiencia que va a consumir. Es en este punto donde dicha orientación al cliente marca la diferencia con respecto a las aproximaciones más convencionales. Ni la consideración del destino como un espacio geográfico y estático donde se producen una serie de relaciones económicas, ni la del destino como un espacio por gestionar desde el punto de vista del marketing para reforzar su atractivo turístico, conciben al turista como un elemento capaz de generar valor añadido al destino a través de su experiencia. El turista ejerce un nuevo rol como productor de experiencias turísticas en el destino (Firat y Dholakia, 2006).

Aparece, por tanto, un nuevo actor en la estructura de los destinos que los DMO (Destination Management Organization) deben considerar en su estrategia de marketing, pues el proceso de gestión de los destinos debe ser ahora más abierto que nunca (García-Rosell y otros, 2007) teniendo en cuenta el potencial que atesoran Internet y las tecnologías de la información y la comunicación (TIC en adelante), especialmente las redes y comunidades en línea, para los destinos turísticos. Se trata de una nueva orientación que Saraniemi y Kylänen (2011) denominan como «social» en cuanto a la construcción de los destinos y productos turísticos. Los destinos concebidos como espacios sociales que se retroalimentan de la información y del conocimiento que aportan los consumidores e incluso sus propios ciudadanos, representan, sin duda, una nueva perspectiva en la visión del concepto de destino. 
Tanto las aportaciones de Saraniemi y Kylänen, especialmente las referentes a una mayor presencia del turista, como las de Pearce, se han visto claramente reforzadas en los últimos años a raíz de la irrupción de las TIC e Internet, en concreto de la web 2.0. Ello ha representando, entre otros, un factor decisivo para que los organismos de gestión de los destinos (DMO en adelante) se hayan visto obligados a redefinir sus estructuras y estrategias de gestión, principalmente en materia de marketing; y dicho ámbito no es el único susceptible de reforzar y retroalimentar su dinámica con la participación de los agentes del destino en este sentido.

En el cometido de los DMO, las TIC, Internet y especialmente la web 2.0 desempeñan un papel clave hoy en día, si se tiene en cuenta el actual contexto de gestión de la información y del conocimiento asociado a la evolución de los destinos y mercados turísticos, y, sobre todo, si se considera el gran potencial que presentan los medios sociales como verdaderas herramientas impulsoras del cambio en la gestión externa e interna de los destinos turísticos. Sin duda alguna, la llegada de la web 2.0 ha abierto la puerta a la participación de todos los agentes del destino en los nuevos sistemas y estructuras de gestión, esto es al desarrollo de un modelo basado en los principios de la gobernanza.

Es en este contexto de integración de las TIC e Internet, de búsqueda de una mejora de la gestión del destino y de la apertura e implicación en dicha tarea de los diferentes agentes del destino y del propio turista, en el que aparece claramente asociado el paradigma de los destinos inteligentes. Se trata, en definitiva, de un nuevo modelo de gestión de los destinos turísticos, en fase de definición e implementación, en el que las TIC e Internet surgen en el centro de todas las gestiones internas y externas que afectan al espacio turístico, con la sostenibilidad y la competitividad de los destinos como principales objetivos de su desarrollo, y con el reto de la gobernanza como uno de los pilares transversales y básicos del nuevo modelo de gestión turística (Invat. tur, 2014).

\section{Las conexiones del paradigma smart con la gestión de destinos turísticos}

En su concepción de la competitividad vinculada a la gestión de las ciudades, Castells (1996) adelanta un elemento que en la actualidad se ha confirmado como esencial para el futuro de las ciudades: la gestión de la información y del conocimiento. En esta línea, Castells y Borja (1997) hacían referencia a que, en la sociedad de la información, la competitividad de las ciudades depende principalmente de tres factores, estrechamente relacionados con el paradigma de la gestión inteligente y perfectamente trasladables al ámbito de los destinos turísticos: conectividad, flexibilidad institucional e innovación.

De la implementación de los factores anteriores, especialmente del último de ellos, subyace un nuevo planteamiento de la estrategia urbana de las ciudades, pues la innovación es considerada por Castells y Borja como la capacidad instalada en una ciudad para generar un nuevo conocimiento, aplicado a actividades económicas y basada en la obtención y el procesamiento de información estratégica. Una nueva orientación en la transformación de las ciudades desde una visión colaborativa y cooperativa entre agentes, más próxima al concepto de "gestión abierta", y a la creación de sinergias y de oportunidades a partir de la información existente y del conocimiento colectivo producido. En definitiva, un giro hacia un modelo de ciudad que optimice la información y el conocimiento, un modelo más «inteligente».

Surge así el paradigma de la inteligencia aplicada a la gestión de las ciudades, contemplado por la Comisión Europea en su estrategia Europa 2020 desde la perspectiva del crecimiento inteligente y asociado al desarrollo de una economía basada en el conocimiento y la innovación (McCann y Ortega, 2011), sin olvidar las vertientes del crecimiento sostenible e integrador (Fernández y González, 2009; Blanco, 2015), y en el que las TIC, especialmente Internet, deben tener un papel clave en la transformación de las ciudades.

Así pues, innovación, tecnología y conocimiento aplicado a la gestión de las ciudades derivan en un marco de desarrollo inteligente (McCann y Ortega, 2011). Su traslado a la gestión del territorio da lugar a la aparición de conceptos como el de «territorio inteligente» y, más tarde, al de smart city o ciudad inteligente, paradigmas de 
gran actualidad entre los nuevos enfoques de la planificación y gestión urbana (Vergara y Rivas, 2004; Fernández y González, 2009; Komninos y Tsarchopoulos, 2013).

Los enfoques sobre el concepto de smart city son múltiples, tantos como ámbitos de la gestión de las ciudades existentes. Pero en prácticamente todos hay una cuestión común: la tecnología como un factor influyente en todos los procesos (Caragliu y otros, 2009; Fundación Telefónica, 2011; Chourabi y otros, 2012). Pero no el único. Pues tal y como señalan Caragliu y otros (2009), existen otros factores clave asociados al proceso de gestión inteligente de las ciudades, como son el capital social, la innovación, la capacidad emprendedora o el desarrollo sostenible en todas sus dimensiones (ambiental, económica y sociocultural).

Desde el punto de vista turístico, el análisis de las implicaciones del paradigma smart city en la gestión de los destinos turísticos conduce al modelo de smart destination o destino turístico inteligente (DTI en adelante), uno de los principales paradigmas del momento. Una clara asociación al turismo de la smart city o ciudad inteligente y reflejo del carácter emergente de las ciudades como centros de conocimiento, gestión de la información, tecnología e innovación (Invat.tur, 2013).

El interés por los DTI coincide con un contexto de cambio estructural en el turismo (impacto de las TIC, nuevos patrones de comportamiento de la demanda, aparición de nuevos modelos de negocio, mayor sensibilidad ambiental, etc.), que requiere nuevos enfoques de gestión, de acuerdo con una serie de objetivos (Ivars, Solsona y Giner, 2016):

- Garantizar la calidad ambiental y la sostenibilidad de los destinos.

- Hallar nuevas vías de competitividad en el escenario turístico actual.

- Enriquecer y diferenciar las experiencias turísticas que ofrecen los destinos.

- Reforzar y comunicar los atractivos del territorio.

- Aprovechar la alta penetración de las TIC en la producción y el consumo turísticos.

Los ámbitos que abarca un DTI son tan amplios como los de la gestión del destino en su dimensión global. Pero su propia concepción alude claramente a la gestión integral del destino y, por ende, basada en un proceso de planificación previo, con una metodología que incluya la participación social y que permita alcanzar el mayor grado de consenso entre los agentes del destino, y al mismo tiempo que tenga en cuenta al turista. Es por ello por lo que un DTI afronta los retos de desarrollar una gestión inteligente a través del establecimiento de mecanismos para la generación de inteligencia colectiva, de ampliar la información y el conocimiento de la demanda y de facilitar el desarrollo de nuevos modelos de negocio.

Uno de los retos principales retos de los DTI es, por tanto, conocer al turista y conocerse a sí mismo, con el fin de poder tomar aquellas decisiones que permitan incrementar la satisfacción del turista y mejorar su experiencia, lo que genera información para convertirla en conocimiento y, de esta forma, mejorar la gestión del destino de forma integral y desde una óptica sostenible que redunde en su competitividad turística.

En este contexto, por una parte subyace la necesidad de que los destinos adopten algunas de las premisas del modelo 2.0, tanto en su vertiente de gestión externa como interna, integrando así en la toma de decisiones a los diferentes agentes, al ciudadano y al propio turista. En la medida en que esto se produzca, los resultados asociados a la generación de inteligencia para la toma de decisiones estratégicas y operativas de los destinos serán cada vez mayores. Y, por otra parte, el objetivo de establecer una conexión real entre el entorno online y el offline a través del uso de herramientas 2.0 entre los protocolos de actuación del destino. Todo ello para prevenir y/o detectar así como monitorizar constantemente lo que sucede en torno al destino en cada momento, especialmente durante la presencia del turista en él. Se trata de que esto repercuta eficientemente en la gestión y en el desarrollo de los destinos y en la propia experiencia final del turista.

Estos dos grandes retos justifican la necesidad de los destinos turísticos de evolucionar hacia un modelo inteligente a partir de un uso de la tecnología que permita mejorar la gestión de la información y del conocimiento sobre su propia evolución, dado que dicha cuestión constituye un factor capital para la competitividad de los destinos en la actualidad, del cual depende la mejora de su oferta, la satisfacción de la demanda y la sostenibilidad de sus recursos, y, en definitiva, la toma de decisiones que afectan a los diferentes ámbitos de su gestión. 


\section{La innovación en los destinos inteligentes y el reto de la gobernanza turística}

La innovación tecnológica, asociada a la utilización de la tecnología como medio para introducir un cambio en las empresas (COTEC, 2015), se vincula en el caso de la gestión de los destinos turísticos a la estrategia de marketing en la mayoría de las ocasiones, sobre todo en su vertiente en línea, pues es el ámbito en el que mayores inversiones y desarrollos tecnológicos se están produciendo. Pero la innovación en la gestión de los destinos no solo se ha producido derivada de la evolución de la tecnología, sino de muchos otros factores vinculados a productos, a servicios, a procesos y a relaciones entre agentes, principalmente.

$\mathrm{Si}$ se trasladan al caso de los destinos turísticos, los diferentes modelos de innovación asociados a las TIC e Internet, todos tienen cierta relevancia. Ahora bien, teniendo en cuenta el incremento del consumo de información en línea y del uso de TIC por parte de la demanda turística, el continuo rediseño del escenario de la distribución turística en línea y las nuevas pautas en las relaciones entre agentes de los destinos y turistas a raíz del uso de nuevas herramientas tecnológicas y medios sociales digitales, es decir, los principales elementos que caracterizan el presente y futuro del panorama turístico, queda claro que los destinos están obligados a integrar la innovación tecnológica a través de las TIC e Internet, principalmente en los siguientes ámbitos de su gestión:

- La planificación y gestión del destino, con el objetivo de alcanzar un nuevo modelo abierto, integrador y dinámico en las relaciones con los agentes clave del destino y los turistas.

- El desarrollo de un nuevo marketing en línea basado en la colaboración y cooperación con el sector y con los turistas, en la vigilancia competitiva, en el desarrollo y adaptación continua de la oferta y en la generación de una inteligencia colectiva que beneficie tanto al destino como a la demanda.

- El fomento y el apoyo a la implementación de políticas de desarrollo turístico sostenible, basadas en el uso de la tecnología tanto en la fase operativa como en la estrategia de la gestión de los destinos.

Por todo lo anterior, los destinos turísticos se enfrentan a un contexto en el que la innovación y la tecnología se sitúan entre los principales ejes sobre los que se sustenta su mejora competitiva. De ello se derivan retos importantes a los que los destinos deben hacer frente, entre los que cabe destacar, por una parte, la mejora de la información y del conocimiento para hacer frente a las exigencias de innovar en el contexto de la «sociedad red»; y, por otra, el impulso a la colaboración y cooperación entre agentes, asociado al concepto de "gestión abierta". En ambos casos se trata de cuestiones que derivan en modelos o paradigmas como el de «destino 2.0» o el de smart destination, que sin duda marcan la referencia del modelo hacia el que los destinos turísticos deben encaminarse, un paso más hacia un modelo colaborativo, participativo y, en definitiva, abierto a los principios de la gobernanza turística e inteligente.

La gobernanza y el modelo DTI se refuerzan mutuamente, de ahí la conveniencia de promoverlos conjuntamente y de una manera integrada (Invat.tur, 2015). Desde el punto de vista del DTI, la gobernanza constituye una de sus características básicas (Giffiger y otros, 2007), centrada fundamentalmente en la participación social, en la toma de decisiones y en la transparencia. Se trata de aspectos fundamentales que facilitan la planificación y la gestión de un destino turístico de forma abierta y colaborativa, a partir de la consideración de las aportaciones del colectivo que forma parte del propio destino turístico y tomando como base el uso de Internet y las TIC.

La inteligencia colectiva de todos los agentes del destino es la que genera el conocimiento y se convierte así en el motor del ecosistema turístico; para ello construye unas nuevas dinámicas que rompen con lo establecido y conocido hasta el momento. Como resultado, un nuevo ecosistema basado en el aprovechamiento del efecto red tanto a nivel interno, en la gestión del mismo y en las relaciones entre los propios agentes que lo conforman, como externo, en las tareas de comunicación y marketing hacia el cliente final (Miralbell, 2010).

Ahora bien, la realidad refleja que, a pesar de que para el caso de los destinos turísticos la integración de TIC y de la web 2.0 representa un elemento de carácter transversal que puede dinamizar y reforzar su estructura interna de relaciones entre los agentes que los conforman (Hays, Page y Buhalis, 2013), y, por tanto, impulsar la optimi- 
zación del efecto red por parte de los destinos, en la mayoría de los destinos turísticos su uso se ha centrado en la vertiente externa de la gestión de la estrategia de marketing y no tanto en potenciar dinámicas de innovación y transferencia de conocimiento a partir de la colaboración entre agentes del destino. Ni las empresas ni las DMO han dado el paso de reforzar su gestión desde la colaboración y la innovación abierta. En ambos casos es probable que ello se deba a la desconfianza habitual que se asocia con la rivalidad entre empresas y organizaciones, junto con los efectos de la brecha digital, tal y como señala Miralbell (2012) como principales barreras y problemas a la hora de adoptar innovaciones tecnológicas para fomentar la colaboración y la generación de conocimiento colectivo.

Los destinos turísticos se encuentran, por tanto, lejos de ser considerados un ejemplo en materia de gobernanza en el marco del modelo DTI, es decir, de aprovechar las facilidades que ofrece el actual contexto de desarrollo tecnológico para obtener un mayor grado de interacción y cooperación entre los actores públicos y privados que garantice una toma de decisiones participativa (Rhodes, 1997; Nátera, 2004). Un aspecto que sin duda alguna supone otro de los grandes retos al que se enfrentan los destinos turísticos.

\section{Conclusiones}

Tras la superación de la fase de expansión del desarrollo de infraestructuras y servicios y de la etapa posterior de dinamización y creación de producto, los destinos turísticos se enfrentan a un nuevo ciclo que les exige reorientar sus estrategias, adoptar nuevas fórmulas de gestión y utilizar nuevos instrumentos.

En este contexto, la tecnología desempeña un papel esencial que puede permitir abordar con mayores garantías los retos que se presentan en el corto, medio y largo plazo para los destinos turísticos. Aunque no se trata del único elemento clave ni de la finalidad del nuevo ciclo de gestión, sino, más bien, de un factor esencial en la consecución de un modelo caracterizado por la inteligencia, la eficiencia y la gobernanza como elementos transversales en las diferentes fases y ámbitos de desarrollo de los destinos en el nuevo paradigma de la gestión inteligente, esto es, del modelo de destino turístico inteligente.

\section{Referencias bibliográficas}

Blanco, J. (2015). Libro blanco de los destinos inteligentes. Estrategias y soluciones para fomentar la innovación en el turismo digital. LID Editorial Empresarial.

Caragliu, A.; Del Bo, CH.; Nijkamp P. (2009). «Smart cities in Europe». Third Central European Conference in Regional Science (CERS). Págs. 45-59.

Castells, M. (1996). The Rise of the Network Society. The Information Age. Economy, Society and Culture, Vol.1. Maldem, MA: Blackwell.

Castells, M.; Borja, J. (1997). Local y global: la gestión de las ciudades en la era de la información. México D. F.: Taurus.

Chourabi. H. y otros (2012). «Understanding Smart Cities: An Integrative Framework». 45th Hawaii International Conference on System Sciences.

Crouch, G. I.; Ritchie, J. R. B. (1999). «Tourism, competitiveness and societal prosperity». Journal of Business Research. N. ${ }^{\circ} 44$, págs. 137-152.

Fernández, J.; González, P. (2009). Evaluación de territorios inteligentes en la sociedad del conocimiento. Netbiblo.

Firat, A.F.; Dholakia, N. (2006). «Theoretical and Philosophical Implications of Postmodern Debates: Some Challenges to Modern Marketing». Marketing Theory. Vol. 6, n. 2 2, págs. 123-62.

Fundación Cotec para la Innovación Tecnológica (2015). «Informe tecnología e innovación en España 2014». Informes sobre el sistema español de innovación.

García-Rosell, J. C. y otros (2007). «From Firms to Extended Markets - A Cultural Approach to Tourism Product Development». Tourism: An International Interdisciplinary Journal. Vol. 55, n. 4, págs. 445-59. 
Hays, S.; Page, S.; Buhalis, D. (2012). «Social Media as a destination marketing tool: its use by national tourism organisations». Current Issues in Tourism. Vol.16, n. ${ }^{\circ}$ 3, págs. 211-239. Disponible en línea [fecha de consulta: 27 de marzo de 2017] <http://dx.doi.org/10.1080/13683500.2012.662215>

Howie, F. (2003). Managing the tourist destination. London: Continuum.

Instituto Valenciano de Tecnologías Turísticas (Invat.tur) (2013). Proyecto destinos turísticos inteligentes Comunitat Valenciana. DTICV.

Ivars, J.; Solsona, J.; Giner, D. (2016). «Gestión turística y tecnologías de la información y la comunicación (TIC): el nuevo enfoque de los destinos inteligentes”. Documents d'Anàlisi Geogràfica. Vol. 62, n. ², págs. 327-346.

Komninos, N.; Tsarchopoulos, P. (2013). «Toward Intelligent Thessaloniki: from an Agglomeration of Apps to Smart Districts». Journal of Knowledge Economy. Vol. 4, n. 2 2, págs. 149-168.

McCann, P.; Ortega, R. (2011). «Smart Specialisation, regional growth and applications to EU Cohesion policy». Economic Geography working paper. University of Groningen.

Miralbell, O. (2010). «Destinos turísticos 2.0. Un nuevo paradigma de gestión y planificación». UOC.

Miralbell, O. (2012). «Webs de xarxes socials i intercanvi de coneixement. Anàlisi de l'adopció i ús dels membres de les comunitats virtuals professionals del turisme» (tesis doctoral). Universitat Oberta de Catalunya (UOC).

Nateral, A. (2004). «La noción de gobernanza como gestión pública participativa y reticular». En: Documentos de Trabajo: Política y Gestión. Universidad Carlos III de Madrid.

Rhodes, R. A. (1997): «Understanding Governance: policy Networks, Governance Reflexivity and Accountability». Buckingham, Open University Press.

Saraniemi, S.; Kylänen, M. (2011). «Problematizing the Concept of Tourism Destination: An Analysis of Different Theoretical Approach». Journal of Travel Research. Vol. 50, n. ${ }^{\circ}$ 2, 133-143.

Vergara, A.; Rivas, J. (2004). Territorios inteligentes. Fundación Metrópoli.

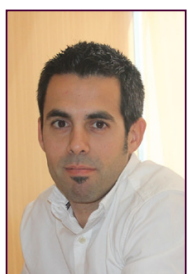

\section{David Giner Sánchez}

Giner_dav@gva.es

Profesor colaborador de los programas de turismo de la UOC. Instituto Valenciano de Tecnologías Turísticas (Invat.tur)

Doctor en Dirección y Planificación del Turismo (Universidad de Alicante) y máster en Planificación y marketing de destinos turísticos (Universitat Autònoma de Barcelona). Codirector del curso de Especialista en big data y turismo de la Universidad Miguel Hernández y profesor colaborador de la UOC desde 2008. Técnico del Instituto Valenciano de Tecnologías Turísticas (Invat.tur) y coordinador, entre otros, del Proyecto destinos turísticos inteligentes Comunitat Valenciana (DTI-CV). Ámbitos de investigación: tecnología, marketing e innovación en la planificación y gestión de destinos turísticos.

Los textos publicados en esta revista están sujetas -salvo que se indique el contrario- a una licencia de Reconocimiento 3.0 España de Creative Commons. Podéis copiarlos, distribuirlos, comunicarlos públicamente y hacer obras derivadas siempre que reconozcáis los créditos de las obras (autoría, nombre de la revista, institución editora) de la manera especificada por los autores o por la revista. La licencia completa se puede consultar en http://creativecommons.org/licenses/by/3.0/es/deed.ca.

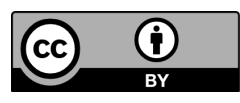

